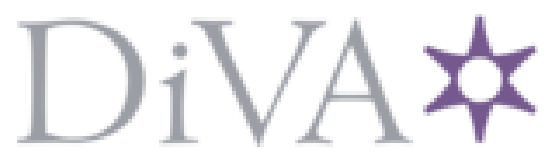

http://www.diva-portal.org

\title{
Postprint
}

This is the accepted version of a paper presented at Position, Location and Navigation Symposium, 05 May - 08 May 2014,USA.

Citation for the original published paper:

Nilsson, J., Rantakokko, J., Händel, P., Skog, I., Ohlsson, M. et al. (2014)

Accurate Indoor Positioning of Firefighters using Dual Foot-mounted Inertial Sensors and Interagent Ranging.

In: Proceedings of the Position, Location and Navigation Symposium (PLANS), 2014 IEEE/ION

N.B. When citing this work, cite the original published paper.

Permanent link to this version:

http://urn.kb.se/resolve?urn=urn:nbn:se:kth:diva- 145354 


\section{Accurate Indoor Positioning of Firefighters using Dual Foot-mounted Inertial Sensors and Inter-agent Ranging}

\author{
J.-O. Nilsson, J. Rantakokko, P. Händel, I. Skog, \\ M. Ohlsson \\ Dept. of Signal Processing, ACCESS Linnaeus Centre \\ KTH Royal Institute of Technology \\ Stockholm, Sweden
}

\author{
K.V.S. Hari \\ Statistical Signal Processing Lab, Dept. of ECE \\ Indian Institute of Science (IISc) \\ Bangalore, India
}

\begin{abstract}
A real-time cooperative localization system, utilizing dual foot-mounted low-cost inertial sensors and RFbased inter-agent ranging, has been developed. Scenario-based tests have been performed, using fully-equipped firefighters mimicking a search operation in a partly smoke-filled environment, to evaluate the performance of the TOR (Tactical lOcatoR) system. The performed tests included realistic firefighter movements and inter-agent distances, factors that are crucial in order to provide realistic evaluations of the expected performance in real-world operations. The tests indicate that the TOR system may be able to provide a position accuracy of approximately two to three meters during realistic firefighter operations, with only two smoke diving firefighters and one supervising firefighter within range.
\end{abstract}

Keywords-Firefighter; localization; foot-mounted INS; ranging; cooperative localization

\section{INTRODUCTION}

A robust indoor localization system is expected to improve the safety and efficiency in firefighting operations. The most important system applications include alarm functionalities, navigation guidance, and enhanced situation awareness. The firefighters main concern is their safety, with particular emphasis on smoke diving (or Breathing Apparatus, BA) operations. The alarm functionality can reduce the risk for firefighters being separated, e.g. by implementing an alarm that activates if the distance between the smoke diving pair increase above a pre-specified distance. A navigation aid can help firefighters to quickly withdraw from dangerous areas, or reduce the time to find and extract firefighters in distress. Finally, the efficiency in firefighting operations can be improved with a situation awareness system which includes information on past positions (trajectories) and rooms/areas that has previously been searched, thereby avoiding double searches of these locations.

Surveys of user requirements and localization technologies are provided in [1-5]. Considering the need for an infrastructure-free localization system and the stringent size, weight, power and cost (SWaP-C) requirements, it is believed that the accuracy and availability requirements can only be fulfilled by embracing a multisensor fusion approach, utilizing sensors with complementary error characteristics [1]. Sensors and localization sub-systems that are being pursued by different research teams include foot-mounted inertial navigation systems (INS) [6-7], back-mounted pedestrian deadreckoning systems [8-11], magnetometers, barometric sensors (using a reference sensor at a known height to counter effects from weather changes), imaging sensors (including visual [12] and thermal infra-red cameras [13]), Doppler radar [14], radiobased ranging [15] using synthetic aperture approaches [16], and cooperative localization approaches [17-18].

A real-time cooperative localization system, utilizing dual foot-mounted low-cost inertial sensors [19] and inter-agent ranging, has been presented in [4,20]. Considering typical deployments and methodology it is conceivable that shortrange ranging transceivers, e.g., based on ultra-wideband (UWB) transceivers, and cooperative localization techniques could be applied in firefighter operations. The distances between the smoke diving pair, and often also to the smoke diving leader, are short enough to allow accurate inter-agent ranging. In large incidents the number of firefighters within range will increase, thereby enabling a higher performance gain by incorporating cooperative localization.

Scenario-based tests are expected to play an important role in the performance evaluations of firefighter positioning systems [21-23], since this ensures that the test subjects use realistic movements and distances between (and number of) firefighters. The former affects the performance of the footmounted INS, especially in operations with heavy smoke and heat that forces the firefighters to move close to the ground. The latter determines the connectivity between the firefighters and thus the possibilities of applying cooperative localization schemes for improved accuracy.

The methodology employed in firefighting operations, determining the relevant test scenarios, varies between countries. Using Sweden as an example, a firefighting team responding to an alarm typically consists of two smoke diving 


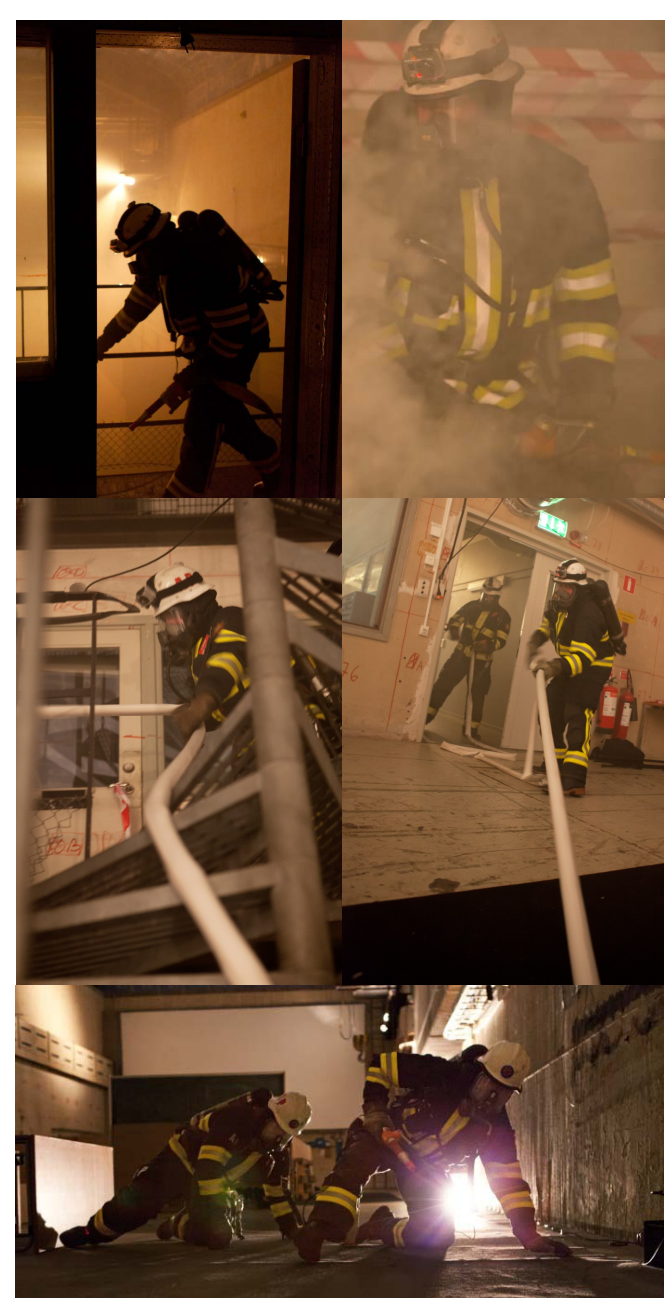

Fig. 1. Typical behavior, movements and firefighter distances during the scenario-based tests that were performed in the KTH R1 facility.

firefighters, one smoke diving supervisor, one firefighter responsible for supplying water, and a sector chief. Typically, two or more of these five-person teams are called in from different fire stations.

Smoke diving operations are performed with firefighters working in pairs with short distances between them and whilst always carrying the water hose. The smoke diving supervisor will stay in a safe area, yet close enough to the smoke diving pair so that he can quickly help them if needed. The smoke diving supervisor should, according to regulations, continuously keep track of the positions and activities of the smoke divers; however, in reality it is extremely difficult to obtain the required information through the radio. The smoke diving supervisor is equipped with breathing apparatus and carries his own water hose, enabling him to aid or rescue the smoke divers if needed. In large incidents, one supervisor can be responsible for multiple smoke diving teams operating within the same area, and replacement or emergency rescue teams can be standing by at the base point prepared for rapid deployment.
Consequently, in this paper we present initial results from scenario-based performance evaluations using the system presented in $[4,20]$. The evaluations were done with a fully equipped smoke diving pair and a smoke diving supervisor. Photographs from the tests are shown in Fig. 1. The results shows that the positioning system using dual foot-mounted inertial sensors and inter-agent ranging could potentially provide the necessary positioning accuracy and availability for smoke diving operations.

\section{DESCRIPTION OF THE TOR SYSTEM}

Each firefighter is equipped with a zero-velocity aided INS $[19,24]$, where tri-axial accelerometer and gyroscope sensors are integrated into the heel of custom-made shoes. Although some of the sensor bias errors for foot-mounted INS can be estimated using zero-velocity updates [25] when the foot is at stand-still, the position and heading error of a foot-mounted INS remains unobservable, and grows (slowly) without bound [4]. Further, the high dynamics of foot-mounted inertial sensors may cause dynamic dependent errors which further worsen the situation. Therefore, to reduce the error growth rate of the individual firefighters (self-contained) positioning modules, the users are equipped with dual foot-mounted INS (one on each foot) and the navigation solution of the two systems are combined. The use of dual foot-mounted INS also increases the robustness towards crawling and other irregular search movements.

Since the human body is non-rigid, the relative positions of the two foot-mounted INS are not fixed, and one cannot directly relate the navigation solution of one foot-mounted INS to another. However, there is an upper limit on how far apart the two foot-mounted INS can be, and the fusion of the two navigation solutions can be viewed as a filtering problem with non-linear inequality constraints [26]. Consequently, no additional measurements are needed to fuse the information from the inertial sensors mounted on different feet.

A centralized cooperative localization algorithm, utilizing the information from UWB-based inter-agent ranging devices combined with the position estimates and uncertainties of each first responder, has been implemented [20]. However, synthetic ranging measurements are generated from position measurements obtained with an Ubisense system (Research \& Development Package), installed in the KTH R1 facility. The performance of the pre-installed Ubisense system has been previously evaluated, see [27]. The TOR system is running in real-time and the firefighter positions are conveyed to all units every second. The fusion algorithm runs in real-time on an Android-based processing platform (Samsung Galaxy S3). The processed data from each firefighter, consisting of position and heading information, is transmitted to the command and control system via IEEE 802.11 WLAN radio links.

Note that even though the cooperation is centralized, the dead reckoning (inertial navigation) is decentralized and hence the system can operate for significant time periods without ranging measurements and contact with the central node. 


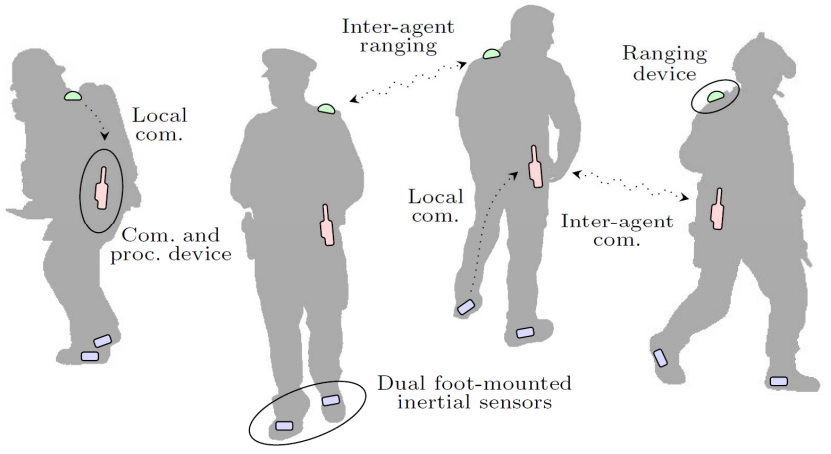

Fig. 2. Illustration of the system architecture of the TOR system, where each agent is equipped with dual shoe-integrated inertial sensor, a processing and communications device, and ranging devices.

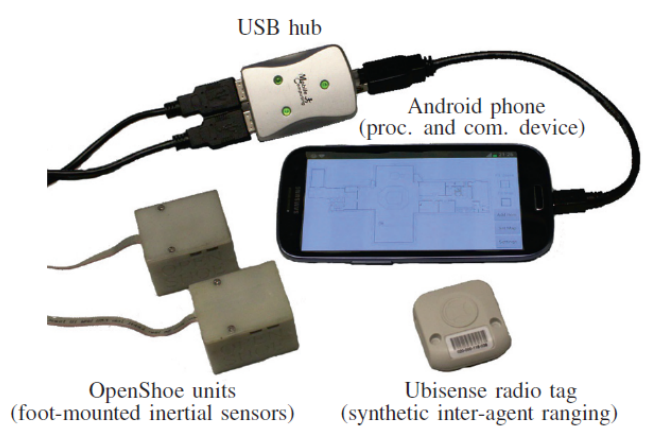

Fig. 3. TOR hardware. Each agent is equipped with two OpenShoe units which are connected to a Samsung Galaxy S3 through cables, and Ubisense radio tags (provides synthetic ranging measurements).

The architecture of the TOR system is illustrated in Fig. 2 and the hardware components are shown in Fig. 3. The footmounted INS, constrained sensor fusion and cooperative localization algorithms are described thoroughly in [20].

\section{FIREFIGHTER TESTS}

Six runs of a simple smoke diving search scenario were performed in the underground KTH R1 facility, using fullyequipped firefighters carrying water hose and nosepiece. Firefighters from two different fire and rescue services were used in the tests; the firefighters from the different stations performed the search using somewhat different movements. The KTH R1 experimental performance space and presence laboratory [28] resembles an old industrial factory, with a large open area (over $300 \mathrm{~m}^{2}$ ), a corridor with rooms, and four stories with several smaller office rooms on each floor on one side of the old nuclear reactor hall. A schematic floor-plan of KTH R1 is shown in Fig. 4, where the approximate positions of the built-up walls used in the tests are marked with red lines.

In the first part of the scenario the pair of smoke-divers searched the upper parts colored in light grey in Fig. 4 whilst walking. They also moved into the corridor to the right and searched the first left room in the corridor, before they returned to the starting position. The second part of the scenario involved searching a heavily smoke-filled area marked with dark grey in Fig. 4. The latter part of the scenario involved

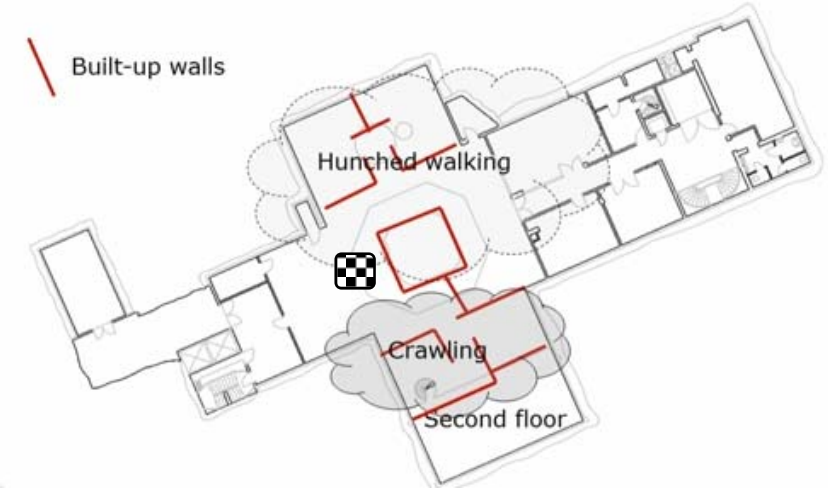

Fig. 4. Schematic of the layout of the KTH R1 facility and the indoor scenario used during the tests. Red lines indicate synthetic walls that were built-up prior to the test. The checker-board area indicates the approximnate start and stop position for the firefighters.

crawling motions and ascending and descending a spiral staircase to the second floor. The first and second parts of the scenario were performed as a consecutive run with the positioning system. The smoke diving supervisor stayed close to the starting point during the tests. The duration of each test was approximately 8-10 minutes.

The position and heading of the firefighters were initialized towards each other and the map through an initialization procedure where the firefighters walked a few times along a line. The cooperative localization algorithm automatically initializes the relative positions and headings of the firefighters as described in [29]; however, the map was manually adjusted to fit the orientation of the firefighters prior to each test.

\section{RESUlts}

Fig. 5-7 shows a representative selection of positioning result from the field tests. Note that all these results are the results from the real-time measurements, without any postprocessing. The magenta and the cyan trajectories and symbols indicate the smoke divers and supervisor, respectively. Fig. 5 shows the result from a run when the system worked without any significant issues. Fig 6 shows a run with the same scenario but performed by firefighters from a different rescue service. The search patterns are different but the performance remains roughly the same. The trajectories follow the true paths well. A video of the results can be viewed at [30].

In the first part of the test the position accuracy is around one meter, as exemplified in the corridor where the door openings were missed by less than a meter. In the second part of the test, where the firefighters mostly crawled while searching the area, the maximum position error is estimated to be between two and three meters. This can be verified for instance by comparing the horizontal positions when the firefighters moved up the stairs with the position of the staircase on the map. Also, the position errors during the second part of the scenario is partially affected by the errors of the first part and small position and orientation errors are expected to remain from the initialization procedure, affecting the performance throughout the whole runs. 


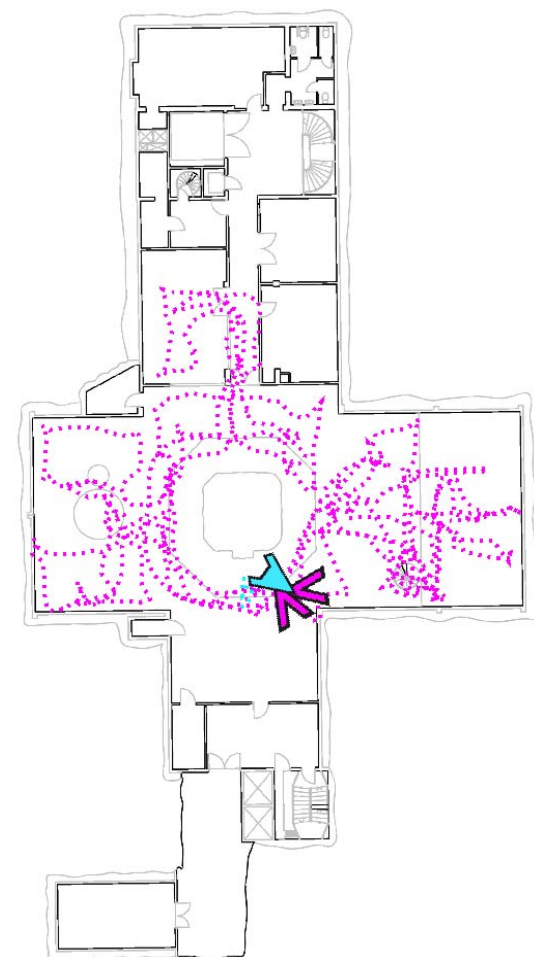

Fig. 5. Test 1 . The smoke divers search the built-up rooms by walking/crawling around the walls of each room. The smoke diving supervisor is passive and waits at the starting position. The positioning system provides a position estimate with a few meters of error throughout the search.

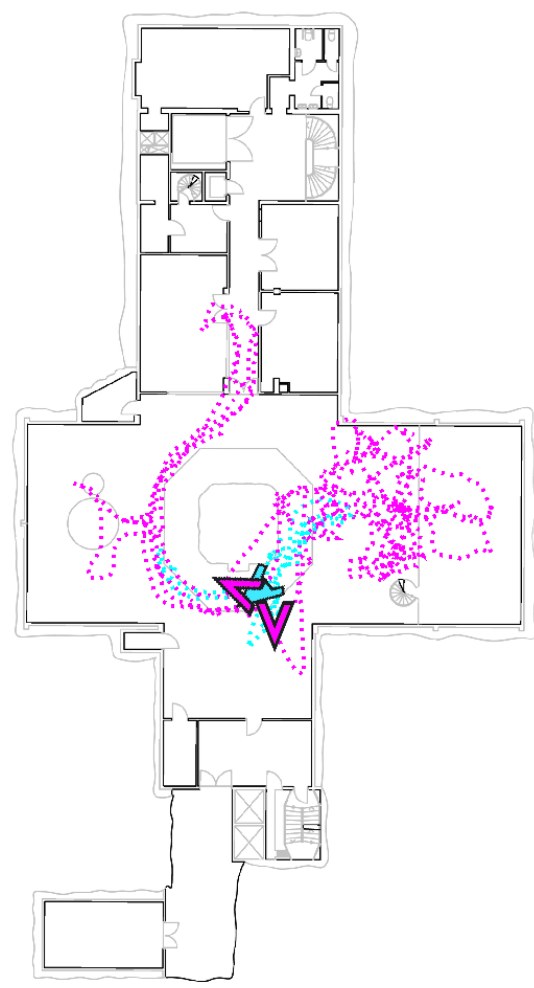

Fig. 6. Test 2. The smoke divers make a quick search of the left and upper side of the site by only entering each room. Thereafter they perform a more thoroughly crawling search of the rooms on the first and second floor of the right side of the site. The smoke diving supervisor follows the smoke divers to help with pulling the hose.

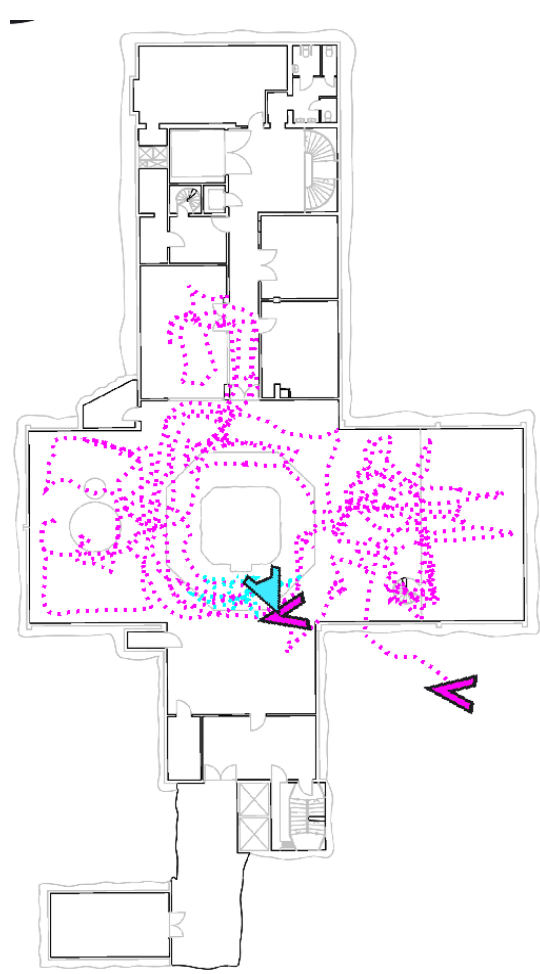

Fig. 7. Test 3 (right). One of the smoke divers experience issues with his system and his heading is thrown off resulting in a large error towards the end. Due to the inconsistency in actual and estimated errors, the system will only slowly recover.

The TOR system is currently a research prototype which has not been hardened to withstand firefighter operations. In several of the tests, one of the firefighters lost connection with one of his foot-mounted units. This resulted in a somewhat worse localization performance but the fact that the system still provides position estimates also highlights the component redundancy provided by the use of dual foot-mounted units. Further, in some of the runs, the crawling motion resulted in lager jumps in the heading. This is likely due to issues with the dual foot-mounted INS integration. However, the exact cause of this is not known. The worst example of this is seen in Fig. 7 where the heading of one smoke diver is thrown off towards the end of the crawling part of the search, giving a large error following the subsequent movement back to the starting position. This is also seen to a lesser extent in Fig 6. The cooperative localization algorithms do not manage to recover the position error during the end of the tests where range measurements were available again (after descending the stairs). This is partially due to the systematic errors not being captured in the uncertainty (error covariance) estimates of the individual firefighter sub-systems, which in combination with robust estimation techniques make the system reject the position of the specific smoke diver. If accurate range measurements were available during a longer period, then the errors would eventually correct themselves slowly.

Two details about the results and setup are worth further mentioning: the synthetic ranging and the tuning of the sensor fusion. The synthetic ranging could lead to overly optimistic results due to avoidance of environmental dependent systematic errors which would arise from ranging being done 
through walls. On the other hand, significant systematic errors are still present and the ranging shows a worse performance compared to commercially available ranging solutions [31-33]. Note that the position errors shown in [27] are from ideal near field conditions (using a horizontal transmitter on a pole) and without differentiation to get range. Consequently, we believe the position results obtained by the TOR system in the evaluations provided herein are not overly optimistic. About the tuning of the system, since these are the first field tests, only rudimentary tuning of the sensor fusion has been done. Especially, no tuning has been done of the foot-mounted inertial navigation systems to accommodate movements other than walking. Also, the embedded inertial sensors (ADIS16367) are of an older generation and better inertial sensors are readily available. This would suggest that the performance results are likely on the pessimistic side.

\section{CONCLUSIONS}

A summary of the position results obtained during scenario-based tests performed with fully-equipped firefighters using the TOR system has been presented. The evaluations indicate that the TOR system could provide sufficiently accurate position estimates in a future firefighter localization system. However, further improvements of the mechanical robustness as well as tuning and adjustment of the sensor fusion algorithms are needed.

The performance of first responder localization technologies is strongly affected by scenario-dependent parameters; hence, scenario-based tests are an important complement in the performance evaluations of these systems.

\section{FUTURE WORK}

First of all, the pre-installed Ubisense system which is currently used to obtain synthetic range measurements will be replaced with time-of-flight measuring UWB transceivers. Both in-house developed UWB transceivers, similar to the systems described in [34], and the Time Domain P410 are being integrated into the system. Secondly, the old inertial sensors are being replaced with wireless and smaller inertial units. In a second iteration these inertial sensors will be replaced with sensors integrated into in-soles that can be easily exchanged when needed [35], as illustrated in Fig. 8. For information on ongoing development see [36].

These upgrades of the TOR system are expected to improve the usability and position accuracy considerably, and continued scenario-based testing, e.g., conducted during regular training exercises, considering various first responder applications will be performed to assess the final performance of the upgraded system.

Finally, exteroceptic sensors, e.g., GNSS-receivers, and the possibility to use (a few) anchor nodes, will be integrated into the system and different initialization schemes suitable for first responder applications will be developed, which perform well both during favorable GNSS conditions and in GNSSchallenged environments.
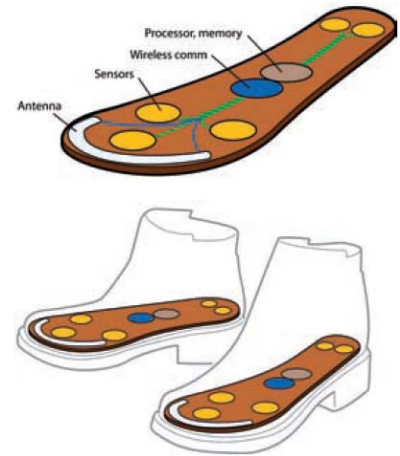

Fig. 8. Illustration of the concept of using removable in-soles equipped with multiple inertial sensors, a processing capability and a wireless body area network communication link to the sensor fusion node on the agent.

\section{ACKNOWLEDGMENT}

We would like to express our gratitude to the firefighters from Uppsala Brandförsvar and Brandkåren Attunda who volunteered to participate in the field trials.

\section{REFERENCES}

[1] J. Rantakokko et al, "Accurate and reliable soldier and first responder indoor positioning: Multisensor systems and cooperative localization," Wireless Communications Magazine, Vol. 18, pp. 10-18, April 2011.

[2] C. Fuchs et al, "Indoor tracking for mission critical scenarios: a survey," Pervasive and Mobile Computing, Vol. 7, pp. 1-15, 2011.

[3] C. Fischer and H. Gellersen, "Location and navigation support for emergency responders: a survey," IEEE Pervasive Computing, Vol. 9, pp. 38-47, 2010.

[4] J.-O. Nilsson, Infrastructure-free Pedestrian localization, $\mathrm{PhD}$ Dissertation, KTH Royal Institute of Technology, Stockholm, Sweden, 2013.

[5] S. Beuregard, Infrastructureless pedestrian positioning, $\mathrm{PhD}$ dissertation, University of Bremen, Germany, 2009.

[6] E. Foxlin, "Pedestrian tracking with shoe-mounted inertial sensors." IEEE Computer Graphics and Applications, pp. 38-46, November/December 2005.

[7] L. Ojeda and J. Borenstein, "Non-GPS navigation for security personnel and first responders," Journal Navigation, Vol. 60, pp. 391-407, 2007.

[8] R. Harle, "A survey of indoor inertial positioning systems for pedestrians," IEEE Communications Survey Tutorials, pp. 1-13, 2013.

[9] www.trxsystems.com

[10] www.seertechnology.com/naviseer

[11] Y. Ma, W. Soehren, W. Hawkinson and J. Syrstad, "Following the team into danger," GPSWorld, June 2013.

[12] M. Veth, Fusion of imaging and inertial sensors for navigation, $\mathrm{PhD}$ dissertation, Air Force Institute of Technology, 2007.

[13] E. Emilsson and J. Rydell, "CHAMELEON on fire - thermal infrared indoor positioning," Proc. of Position Location and Navigation Symposium (PLANS), Monterey, CA, May 2014.

[14] W. Hawkinson, P. Samanant, R. McCroskey and R. Ingvalson, "GLANSER: Geospatial location, accountability, and navigation system for emergency responders - system concept and performance assessment," Proc. of Position Location and Navigation Symposium (PLANS), Myrtle Beach, SC, April 2012.

[15] K. Pahlavan, X. Li, and J.-P. Mäkelä, "Indoor geolocation science and technology," IEEE Communications Magazine, Vol. 40, 2002.

[16] A. Cavanaugh, M. Lowe, D. Cyganski and R.J. Duckworth, "WPI precision personnel locator: Inverse synthetic aarray reconciliation tomography performance," Proc. of ION International Technical Meeting (ITM), January 2013. 
[17] A. Kealy et al, "Collaborative navigation with ground vehicles and personal navigators," Proc. of Indoor Positionind and Indoor Navigation (IPIN), Sydney, Australia, November 2012.

[18] H. Wymeersch, J. Lien, and M. Win, "Cooperative localization in wireless networks," Proceedings of IEEE, 2009.

[19] J.-O. Nilsson, I. Skog, P. Händel, and K.V.S. Hari, "Foot-mounted inertial navigation for everybody - an open-source embedded implementation," Proc. of Position Location and Navigation Symposium (PLANS), Myrtle Beach, SC, April 2012.

[20] J.-O. Nilsson, D. Zachariah, I. Skog and P. Händel, "Cooperative localization by dual foot-mounted inertial sensors and inter-agent ranging," EURASIP Journal on Advances in Signal Processing, 2013.

[21] J. Rantakokko, E. Emilsson, P. Strömbäck and J. Rydell, "Scenariobased evaluations of high-accuracy personal positioning systems," Proc. of Position Location and Navigation Symposium (PLANS), Myrtle Beach, SC, April 2012.

[22] J. Rantakokko, Test Methodology for Evaluations of Soldier Positioning Systems, FOI Report, FOI-R--3819--SE, December 2013.

[23] M. Harris, "The Way Through the Flames," IEEE Spectrum, vol. 9, pp. 26-31, 2013.

[24] I. Skog, P. Händel, J.-O. Nilsson and J. Rantakokko, "Zero-velocity detection - an algorithm evaluation," IEEE Trans. on Biomedical Engineering, Vol. 57, pp. 2657-2666, 2010.

[25] C. Jekeli, Inertial Navigation Systems with Geodetic Applications, de Gruyter, Berlin, 2001.

[26] I. Skog, J.-O. Nilsson, D. Zachariah and P. Händel, "Fusing information from two navigation systems using an upper bound on their maximum spatial separation," Proc. of International Conference on Indoor
Positioning Indoor Navigation (IPIN), Sydney, Australia, November 2012.

[27] A. De Angelis, P. Händel, and J. Rantakokko, "Measurement report: Laser total station campaign in KTH R1 for Ubisense system accuracy evaluation," Tech. Report, KTH Royal Institute of Technology, 2012. QC20120618.

[28] KTH R1 Experimental Performance Space and Presence Laboratory www.r1.kth.se

[29] J.-O. Nilsson and P. Händel, "Recursive Bayesian initialization of localization based on ranging and dead reckoning," Intelligent Robots and Systems (IROS), 2013 IEEE/RSJ International Conference on, Tokyo, Japan, Nov 2013.

[30] http://youtu.be/Qtyq86tJrZ8

[31] www.timedomain.com

[32] D. Dardari, A. Conti, J. Lien and M. Win, "The Effect of Cooperation on UWB-Based Positioning Systems Using Experimental Data," EURASIP Journal on Advances in Signal Processing 2008, 2008:513873

[33] S. Huseth, B. Dewberry, R. McCrosky, "Pulsed-RF Ultrawideband Ranging for the GLANSER GPS-Denied Emergency Responder Navigation System," Proc. of the 2011 International Technical Meeting of The Institute of Navigation, San Diego, CA, January 2011.

[34] A. De Angelis, S. Dwivedi and P. Händel, "Characterization of a flexible UWB sensor for indoor localization," IEEE Trans. On Instrumentation and Measurements, Vol. 62, pp. 905-913, 2013.

[35] K.V.S. Hari, J.-O. Nilsson, I. Skog, P. Händel, J. Rantakokko and G.V. Prateek, "A prototype of a first responder indoor localization system," Journal of the Indian Institute of Science, Vol. 93:3, pp. 511-520, 2013.

[36] http://www.openshoe.org 